\title{
Aerothermoelastic Simulation of Air-Breathing Hypersonic Vehicles
}

\author{
Ryan J. Klock ${ }^{*}$ and Carlos E.S. Cesnik ${ }^{\dagger}$ \\ University of Michigan, Ann Arbor, MI 48109-2140
}

\begin{abstract}
This paper describes the development and implementation of a partitioned-based, multiphysics, multi-fidelity simulation framework for flexible hypersonic vehicles. This simulation framework will enable the study of the dominant physics needed for the generation of control-oriented models and will provide a reference model for flight control evaluation. A partitioned-based solution is employed to approach the problem of modeling a flexible hypersonic vehicle by dividing the vehicle into discrete regions within which unique combinations of physical processes are relevant. The dominant physics of each region are then modeled locally and information is exchanged across region interfaces at predetermined time intervals as the vehicle simulation is marched forward in time. The highly coupled physical processes within each region are modeled using an array of variable fidelity reduced order models. The partition solution implementation is compared to a monolithic solution through trim and time simulation of a sample hypersonic vehicle geometry. Trim results of rigid and flexible vehicle models show good matching between the partitioned and monolithic solutions for Mach $6,26 \mathrm{~km}$ altitude, steady level flight. Time simulations at the same flight conditions also show good qualitative matching between the solutions, but a mismatch in effective mass distribution allows the monolithic vehicle model to have a slightly faster control response to a commanded elevon deflection. The partitioned solution code architecture is then extended to the characterization of flutter for a hypersonic lifting surface, showing a significant reduction of flutter Mach number as a function of flight time.
\end{abstract}

\section{Introduction}

$\mathrm{H}$ YPERSONIC flight presents highly coupled physical processes that must be adequately modeled in order to design the vehicle and its flight control laws. Unsteady aerodynamics, structural dynamics, aerothermal heating, thermal degradation of material properties, geometric stiffening due to thermal gradients, and thermochemical processes all play integral roles in the performance of a hypersonic vehicle (HSV). Due to these coupled and generally complex processes, the reduction of HSV states to manageable numbers has been a daunting task and posed as a significant hurdle to the timely evaluation of vehicle response and stability.

Past research by Bolender and Doman ${ }^{1}$ described a two-dimensional longitudinal flight dynamics model which employed a combination of oblique shock, Prandtl-Meyer expansion, and a quasi-one-dimensional duct with heat addition to determine the stability characteristics of a two-dimensional HSV. The inclusion of shock-expansion theory, rather than the previously studied ${ }^{2}$ Newtonian impact theory, allowed for the consideration of engine inlet spillage and inlet shock patterns which are both considered with respect to a movable inlet door intended to maintain a shock-on-lip condition. Pressures on the aft body resulting from the propulsion exhaust are also considered. Flat plates are used to approximate control surfaces positioned near the tail of the vehicle, which also employed shockexpansion theory in order to determine lift and drag characteristics during simulation. Vehicle flexibility was approximated by a pair of cantilevered beams, one reaching fore and the other aft, joined at the center of mass of the vehicle. The frequency response of the joined-beam model was then analyzed to determine the main vibrational modes of the beam structure and used to generalize the equations of motion for the vehicle. The equations of motion were linearized about a trim condition and used to show unstable short-period and phugoid modes. Bolender and Doman $^{1}$ concluded that research into a closed-loop control system would be required. However, consideration of only two-dimensional flight limited the development of HSV control laws which would ultimately be required to

\footnotetext{
* Graduate Research Assistant, Department of Aerospace Engineering, rjklock@umich.edu

${ }^{\dagger}$ Professor, Department of Aerospace Engineering, AIAA Fellow, cesnik@umich.edu
} 
operate in full three-dimensional, six-degree-of-freedom environments where both longitudinal and lateral flight characteristics must be considered.

In order to explore the characteristics of a three-dimensional vehicle flight, Frendreis et al. ${ }^{3}$ have conducted a full six-degree-of-freedom analysis of a generic hypersonic vehicle which included a rigid vehicle structure, twodimensional shock expansion theory for the external panel pressures, and a one-dimensional area ratio model of the propulsion system. This work was then expanded by Falkiewicz, Frendreis, and Cesnik ${ }^{4}$ to include the effects of a flexible fuselage, flexible control surfaces, the resulting inertial coupling, unsteady aerodynamics, and aerothermal effects by partitioning the HSV into discrete component regions among which information was exchanged to maintain vehicle continuity. This approach was and will be referred to in this paper as the HSV partitioned solution. The work by Falkiewicz et al. ${ }^{5,6}$ focused on enhancing the fidelity of the HSV control surfaces by considering the unsteady aerodynamics, aerothermal heating, and the resulting material property degradation of the control surface structure by way of structural Ritz modes and proper orthogonal decomposition (POD) of the dominant thermal modes. Meanwhile Frendreis and Cesnik ${ }^{7}$ focused on the application of vehicle flexibility to the three-dimensional model by a modal representation of the fuselage deformations. Work by Dalle et al. ${ }^{8}$ applied the two-dimensional MASIV scramjet propulsion model to three-dimensional flight of a rigid HSV based on Ref. 7 to determine vehicle trim. Further work by Dalle and Driscoll ${ }^{9}$ eventually lead to the continuous differentiation of a HSV system for flight trajectory simulation, stability analysis, and model parameter sensitivity analysis for a simple rigid sixdegrees-of-freedom on an ellipsoidal Earth.

The aim of this paper is to further expand the work by Falkiewicz, Frendreis, and Cesnik ${ }^{4}$ and bring together many of the previously mentioned reduced order model (ROM) analyses into a single HSV partitioned solution simulation framework.

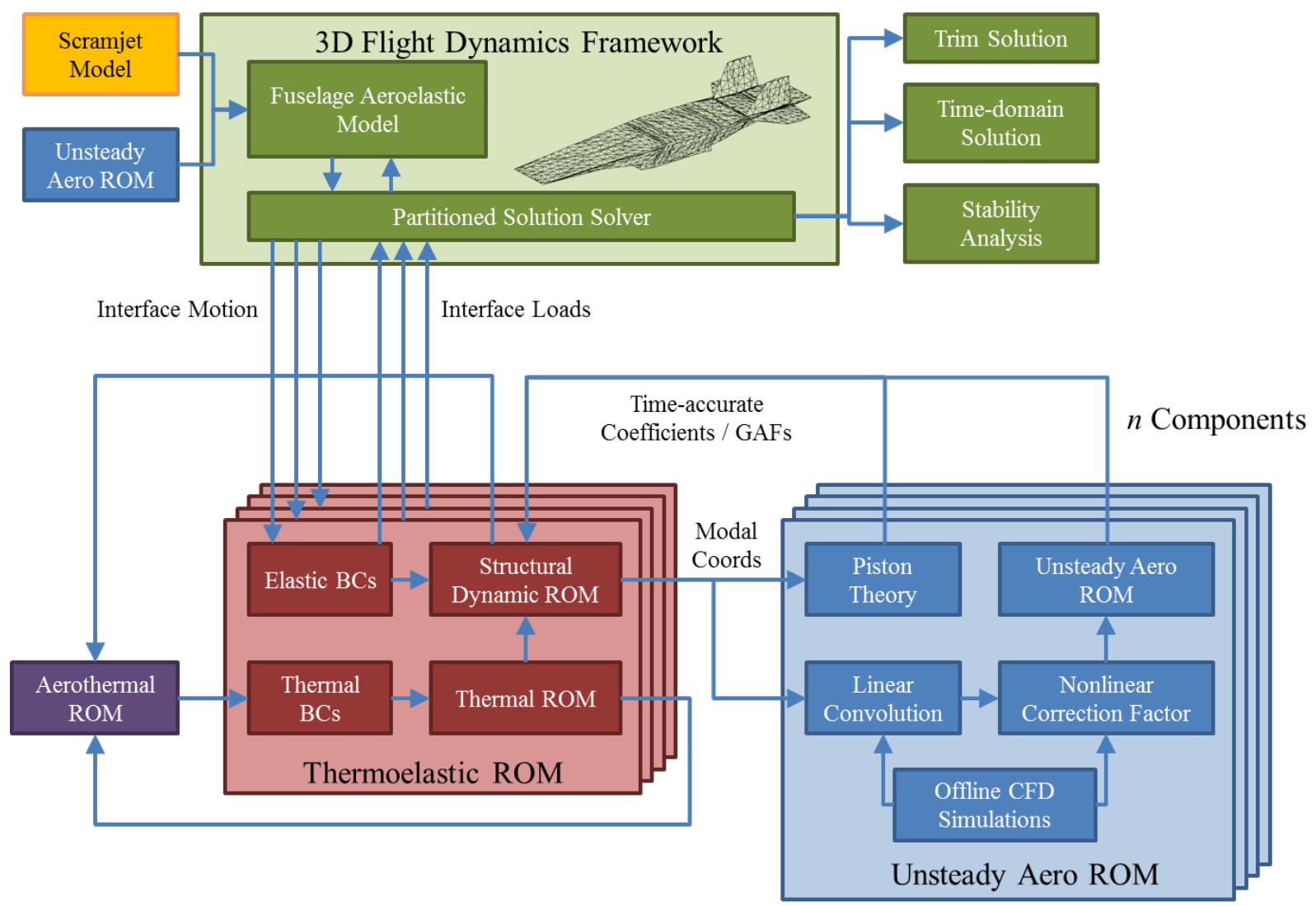

Figure 1: Block diagram of the partitioned solution approach

\section{Theoretical Development}

To effectively bring together a wide spectrum of numerical models, each focusing on a particular portion of a HSV, the partitioned solution approach has been developed. With this approach, the HSV is divided into $n$ components of 
interfacing models as shown in Fig. 1. Each model is a self-contained system of equations tailored to capture the relevant related physics. Each region's models are integrated in time and at every system time step, information between regions in exchanged across interfaces. An example of such physical partition is shown in Fig. 2 with more details of the partitioned regions given in following sections.

There are several advantages to the partitioned solution simulation over a single monolithic simulation. Firstly, entire regions of the HSV may be exchanged, removed, or isolated without affecting the operability of the others. This enables fast trade studies of various component types and models of varying fidelity. Different phenomena such as divergence or flutter of a lifting surface, shock interaction in the propulsion system, or a multitude of other possible problems can be analyzed within the same simulation architecture.

Secondly, physical processes that are unimportant at a particular region need not be modeled. Alternatively, processes that are the primary performance drivers for other regions may be emphasized through the use of higher fidelity models or finer discretization. This reduces computational cost while increasing overall HSV model fidelity.

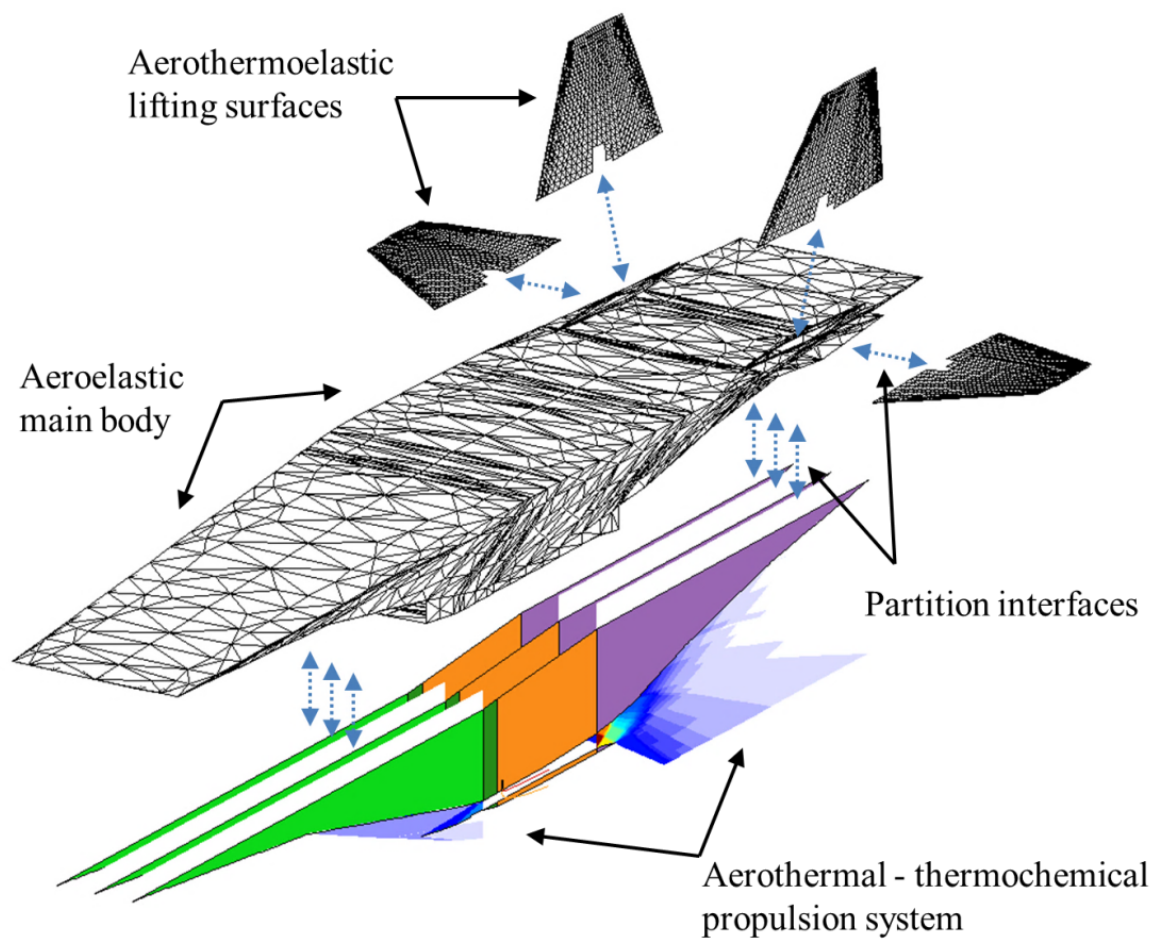

Figure 2: Graphical layout of sample partitioned HSV regions

\section{A. Aeroelastic Main Body}

For a typical cruiser HSV there may exist three types of regions. The first is the main body which is often the main feature of a HSV and provides a vehicle frame of reference, not necessarily the vehicle's center of mass. For the purposes of this paper, we will consider the HSV main body to be a flexible structure subject to unsteady aerodynamic forces over its outer mold line (OML), interfacial loads with other adjoined regions, and the influence of gravity. Given that the main body is often significantly thermally protected since it houses the flight control electronics, fuel supply, and payload, it is assumed that the thermal effects in the structural dynamics are negligible. This is by no means a theoretical restriction of the partitioned solution approach, but rather a choice made to ease later implementation.

\section{B. Aerothermoelastic Lifting Surfaces}

The lifting surfaces of a HSV are typically thin structures experiencing strong heat from the flow. Due to these conditions, the processes of unsteady aerodynamics, aeroheating, material degradation, thermal stresses, and structural dynamics are all relevant to its performance. For the lifting surface to main body interface, the main body exchanges structural displacements and velocities at the root and the lifting surface feeds back root forces and 
moments in response to its motion. For the purposes of this paper, we have considered the interface of the HSV main body to the lifting surface to be a perfectly rigid torque tube which applies the motions of the main body root as a boundary condition to the structural nodes within a lifting surface. The combination of aerodynamic, thermal, inertial, and elastic loads are then used to determine the response of the lifting surface. The lifting surface is considered to act as an all-movable control surface for the HSV actuated by the rigid torque tube. No gap effects are taken into account.

\section{Aerothermal - Thermochemical Propulsion System}

The final region type considered here is that of the air-breathing ram/scramjet propulsion system. This is the underside of the main vehicle body. Such engines consist of four distinct sub-regions. The oncoming flow first encounters the inlet ramp which imposes a series of oblique shock waves that serve to compress the flow. In the case of a ramjet, the flow then enters the isolator. The isolator imposes an oblique shock train which further compresses the flow before terminating in a normal shock. In the case of a scramjet, the isolator is combined with the inlet ramp and does not terminate the supersonic flow with a normal shock, but instead permits a supersonic flow to proceed throughout the engine. The flow then enters the combustor, where fuel is rapidly mixed and ignited before being expelled into the nozzle. The nozzle expands the often still reacting flow, producing thrust. Due to the short residence time of any given portion of flow within the propulsion system, the system is typically as long as possible and encompasses much of the length of the HSV, defining the shape of the main body. The interface between the main body and propulsion system is therefore relatively intimate when compared to the interface between the main body and lifting surfaces. For the purposes of this paper, the deformable main body imposes the flow path geometry for the inlet ramp and nozzle as well as the flight conditions in terms of Mach number, altitude, and angle of attack. The propulsion system returns the thrust, lift, moments, and the pressure distribution on the underside of the main body.

\section{Numerical Implementation}

The publish-subscribe (PS) code architecture is adopted to implement the partitioned solution approach. In PS architecture each function sends and receives relevant data through communication with a central data manager. An individual function does not require knowledge of how the information was first published to the data manager, but only that the information is available for subscription. After processing, each function then publishes its results back to the data manager for use by other functions. Since no data is specifically sent between high level functions, functions may be added, exchanged, or removed without significant impact on the operation of other code processes. This permits one to freely exchange the number and arrangement of components and models used during simulation and process a wide variety of different HSV geometries and simulation fidelities without rewriting any code. For the purposes of this paper, the primary physics models were sorted by component region, with the root displacements, main body OML geometry, HSV flight conditions, and generalized forces held within the central data manager. A high level overview of the code layout is shown in Fig. 3.

Each of the dark process blocks outlined in Fig. 3 represents either a process model or data class that is selfcontained and serves the respective system of models outlined by the lighter partitioned regions. These models may be exchanged for any of a number of models which seek to capture similar physical effects. Each model or partitioned region also is free to sub iterate between communication with the central data manager, allowing the user or function to regulate the discretization time, space, or other parameter dimensions to maximize fidelity or reduce computational cost for particularly demanding functions. 


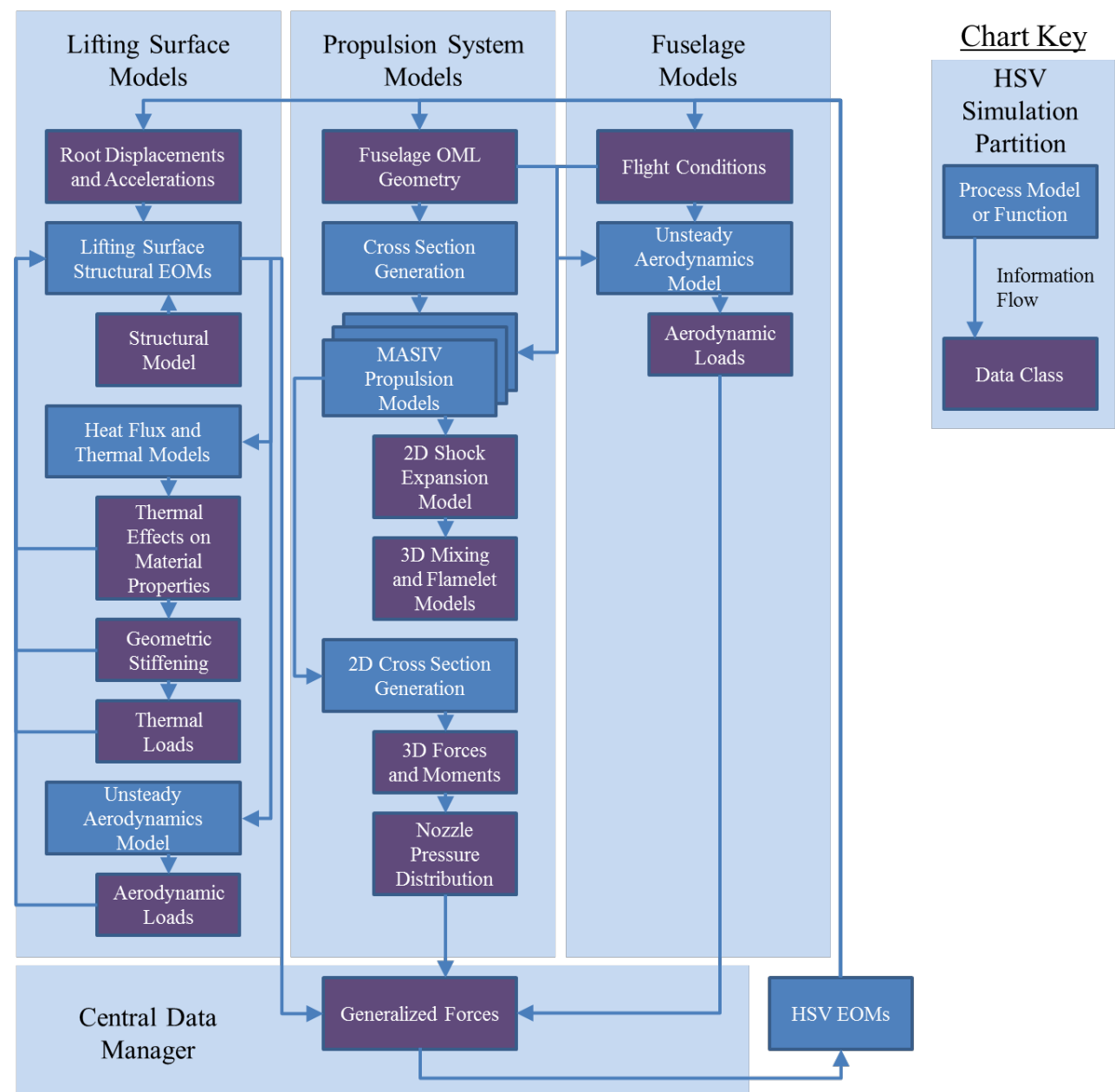

Figure 3: High level overview of HSV simulation code architecture

\section{A. Flexible Vehicle Equations of Motion (EOMs)}

To reduce the number of elastic degrees of freedom present in the HSV main body during three-dimensional flight a modal approach is used. ${ }^{8}$ The HSV EOMs may be expressed in matrix form as

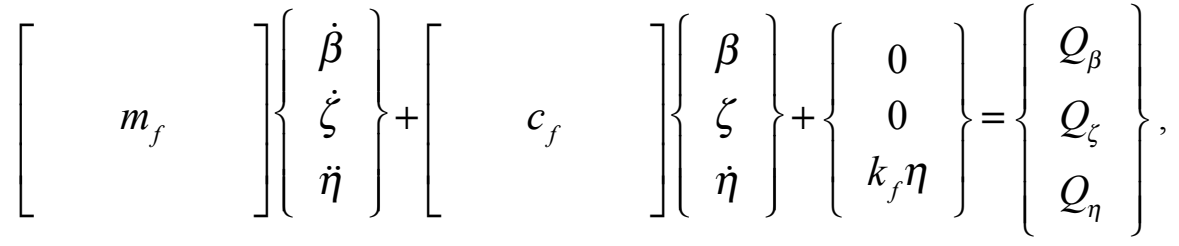

where $\beta$ represents the three orthogonal rigid body velocity components of the body reference frame with respect to the inertial Earth, $\zeta$ represents the corresponding three rigid body rotational velocities, $\eta$ represents the structural deformations expressed in modal coordinates, $m_{f}$ is the generalized mass matrix, $c_{f}$ is the generalized damping matrix, and $k_{f}$ is the generalized stiffness matrix. In order to integrate Eq. 1 for $\beta, \zeta$, and $\eta$, we must determine the generalized forces $Q_{\beta}, Q_{\zeta}$, and $Q_{\eta}$. These generalized forces may come from any of four external forces due to aerodynamics forces on the OML, gravitational forces, interfacial forces and moments from the lifting surfaces, and interfacial forces and moments from the propulsion system.

For the purposes of this study, we have taken the main body to have three modes: lateral bending, longitudinal bending, and torsion based on a free-free Euler-Bernoulli beam. Selection of these modes was described by 
Frendreis and Cesnik. ${ }^{7}$ It is important to note that any set of mode shapes may be implemented and this approach is not limited to the modes we have chosen for this particular study.

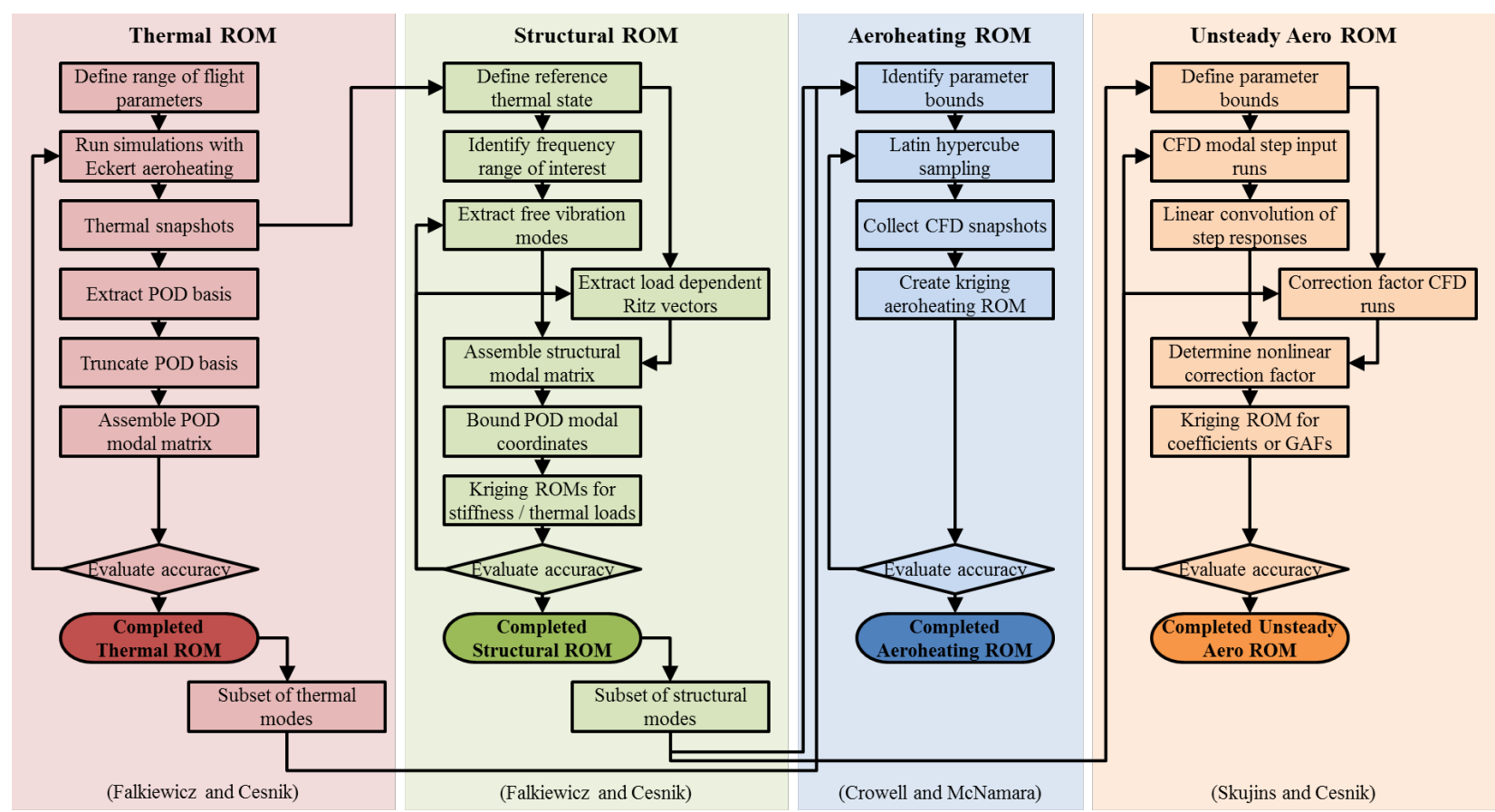

Figure 4: Flowchart overview of the thermal, structural, aeroheating, and unsteady aerodynamics ROM generation processes for an HSV lifting surface

\section{B. Thermal and Aeroheating Models}

The thermal state of the lifting surface structure was modeled using a 32 POD modal basis developed by Falkiewicz and Cesnik. ${ }^{6}$ In their study, the Eckert reference enthalpy method ${ }^{15}$ and a high fidelity finite element structural model was used to create a set of thermal state snapshots which in turn were used to create the POD basis for the transient thermal state of the lifting surface. An overview of the thermal ROM generation is shown in Fig. 4 .

A kriging ROM of the Stanton number was later created as part of Crowell and McNamara's ${ }^{16}$ comparison of reduced models for computational aerothermodynamics and involved a Latin hypercube sampling of aeroheating rates determined from CFD runs. Each CFD run was created by superposition of the thermal and structural modes previously determined by in Ref. 6. Description of the thermal, aeroheating, and structural ROMs integration is given in Ref. 5.

For simulation of the HSV, both the Eckert reference enthalpy method and the Stanton number kriging ROM are available for determining the heat flux to the lifting surface. Additional heat flux and thermal modals beyond those described here may also be used in the partitioned solution but have not yet been implemented.

\section{Lifting Surface Structural Model}

Each lifting surface contains an independent set of EOMs. For the sake of brevity, we will not describe these EOMs here, however the development and discretization of these EOMs is described by Falkiewicz et al. ${ }^{4}$ In order bring the main body and lifting surfaces into equilibrium, an iterative approach also described by Falkiewicz et al. ${ }^{4}$ is used, but expanded to include both $x$ - and $z$-direction root motion imposed by the main body. Motion along the $y$ direction is assumed to have little effect on the dynamics of the lifting surface and it is neglected. As with the main body, the number of degrees of freedom of the solution is reduced through a modal approach. The number and shape of the modes are arbitrary and entered by the user. For the analysis presented here, a set of 15 Ritz modes composed of the first 10 free vibration modes and 5 static load shapes is used as described by Falkiewicz. ${ }^{19}$ An overview flowchart of this ROM generation process is shown in Fig. 4. The first five free vibration mode shapes are given in Fig. 5. 

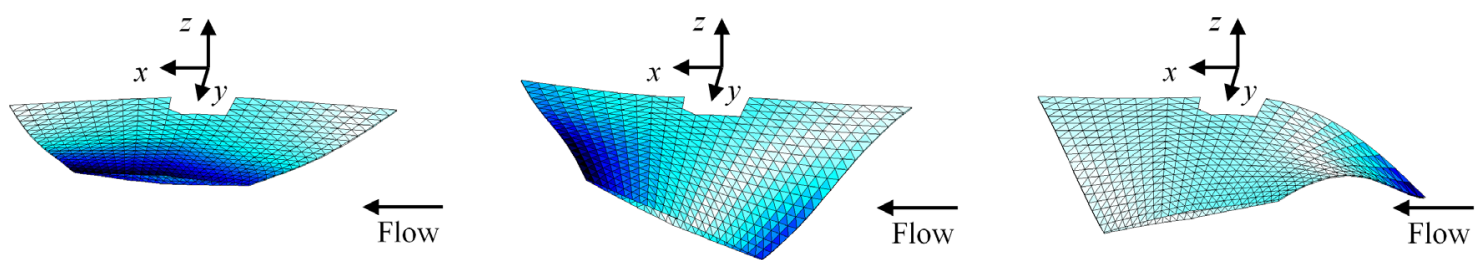

a. Mode 1: $25.1 \mathrm{~Hz}$

b. Mode 2: $35.6 \mathrm{~Hz}$

c. Mode 3: $53.2 \mathrm{~Hz}$
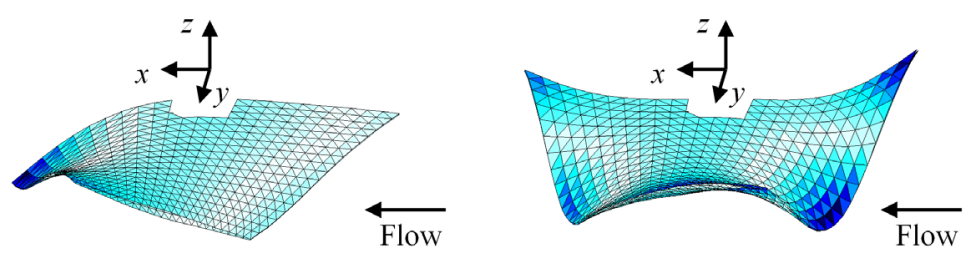

d. Mode 4: 54.3 Hz

e. Mode 5: $86.1 \mathrm{~Hz}$

Figure 5: Lifting surface first five structural free vibration modes

\section{Unsteady Aerodynamics Models}

To model the aerodynamic pressures on the OML of the HSV, an iterative approach is applied in which the OML pressure distribution is recalculated at discrete aeroelastic time steps. The current aerodynamic models available to calculate the pressure distribution of the main body and lifting surfaces are the conventional steady shock and Prandtl-Meyer expansion model, ${ }^{10}$ shock-expansion with a third-order piston theory correction term, ${ }^{13}$ Newtonian scalar pressure model, ${ }^{12}$ Newtonian vector pressure model, ${ }^{12}$ and a purely third-order piston theory model. ${ }^{13}$ It is important to note that piston theory is limited to normal body motions slower than the speed of sound. An additional unsteady aerodynamics model is available to the lifting surfaces through the work of Skujins and Cesnik $^{14}$ in which linear convolution combined with a nonlinear correction factor are extracted directly from CFD runs as outlined in Fig. 4.

\section{E. Propulsion Model}

The Michigan-AFRL Scramjet-in-Vehicle (MASIV) propulsion model by Torrez et al. ${ }^{17}$ is a $2 \mathrm{D}$ supersonic flow and combustion solver designed for the evaluation of ram and scramjet propulsion systems. A sample scramjet flow path and hypersonic flow pattern is shown in Fig. 6. The 2D flow patterns in the inlet and nozzle sections are solved using the method of characteristics with the fundamental quantities of shock waves, expansion waves, vehicle surfaces, and contact discontinuities. When two wave fronts are found to intersect, a Riemann problem is solved which produces two new waves and a slip line. Within the combustor section, a quasi-1D technique is used with 3D mixing and flamelet chemistry for either hydrogen or ethylene fuel.

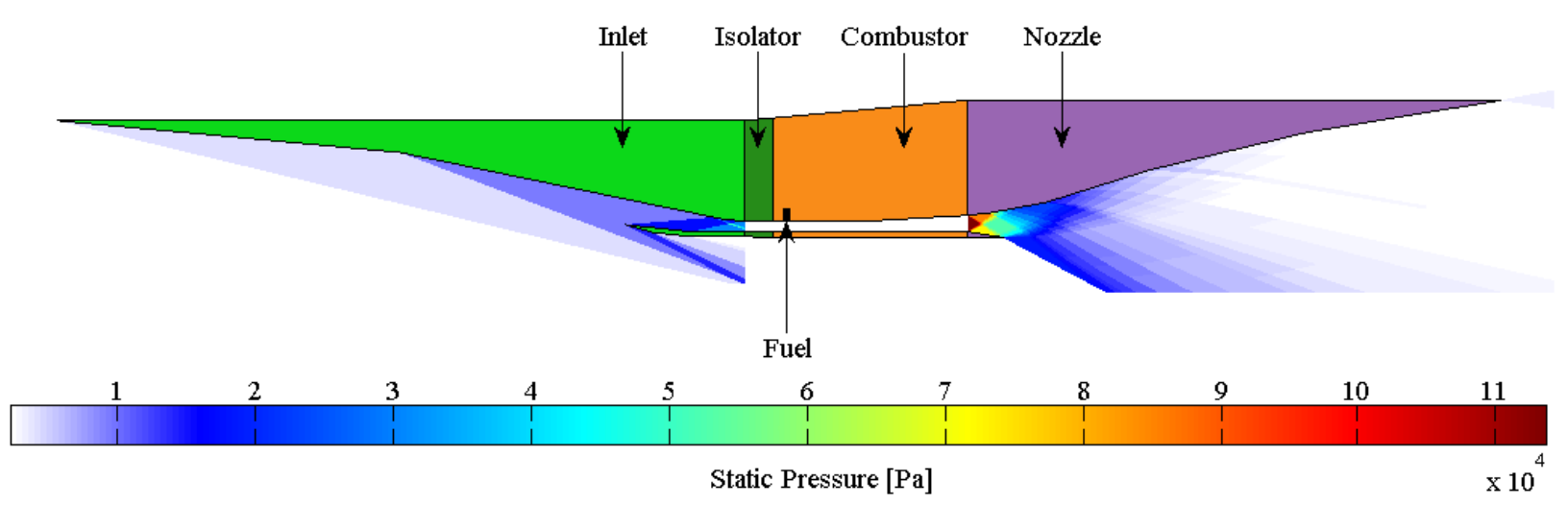

Figure 6: Sample scramjet flow path and hypersonic flow pattern, $-0.6^{\circ}$ angle of attack, Mach $6,26 \mathrm{~km}$

7 of 16

American Institute of Aeronautics and Astronautics 
To accommodate a 3D propulsion model using the 2D MASIV code, multiple 2D engine strips along the main body are used. Each cross section is then used to construct an engine flow path which is passed to the MASIV code. Using this approach, it is assumed the engine flow path does not vary greatly along the vehicle span. Due to the flexible nature of the main body and its influence on the inlet and nozzle geometry there will be some variation along the engine span. By considering the engine through a number of sample cross sections and interpolating each sectional performance, these effects can be captured approximately.

After processing the propulsion cross sections, the quantities of sectional thrust, lift, moment, and pressure distribution are extracted from the MASIV solution. Each thrust and lift force is crossed with the moment arm of the propulsive model reference centroid to the origin of the main body reference system to account for moments. The resulting force and moment distributions are fit to a quadratic least square regression along the main body span to smooth small non-uniformities which arise from the sensitivity of MASIV to distortion of the grid cells due to the main body deformation. The pressure distribution along each cross section is similarly interpolated and applied to the surface of the main body corresponding to the nozzle. Due to the way the main body is partitioned into upper and lower parts, there is a guarantee distinction of the source of forces and moment coming from the engine versus the main body aerodynamics, avoiding any double counting of pressure forces.

\section{Numerical Results}

In what follows, a sample HSV model is given. To verify that the partitioned solution accurately represents the characteristics of a sample vehicle, the HSV model is represented in two forms. The first form is the vehicle partitioned as described in the numerical implementation section. The second form is a monolithic representation of the same HSV as a single main body object subject to elastic deformation and unsteady aerodynamics. Deflections of the lifting surfaces are expressed by modifying the OML of the main body whenever there is a commanded change. Elastic deformations of the lifting surfaces are expressed by the same lateral, longitudinal, and torsional modes as the fuselage. The partitioned and monolithic solutions are compared through their respective trim and time solutions.

An initial study of the lifting surface flutter margin based on flight trajectory and time of flight is considered. The surface structure is exposed to aeroheating at various Mach numbers and for various durations. The frequency and damping of the 15 modes described in section III. C. are determined through the eigenvalues of a linear state matrix identified from mode specific perturbations to a time simulation. Flight times and Mach numbers corresponding to eigenvalues with zero real parts represent the flutter boundary.

\section{A. Basic Vehicle Model}

A sample vehicle's structural properties and configuration are shown in Table 1 and Fig. 7, respectively.

Table 1: Sample HSV structural parameters

\begin{tabular}{ccc}
\hline Parameter & Description & Value \\
\hline$L_{f}$ & Forebody length & $15.2 \mathrm{~m}$ \\
$L_{a}$ & Aftbody length & $15.2 \mathrm{~m}$ \\
$\mu_{f}$ & Forebody mass / unit length & $8.38 \times 10^{2} \mathrm{~kg} / \mathrm{m}$ \\
$\mu_{a}$ & Aftbody mass / unit length & $1.25 \times 10^{3} \mathrm{~kg} / \mathrm{m}$ \\
$I_{x}$ & length & $3.28 \times 10^{3} \mathrm{~kg} \mathrm{~m}{ }^{2} / \mathrm{m}$ \\
& & \\
$E I_{z z}$ & Rotational inertial about the vehicle length / unit & $5.06 \times 10^{10} \mathrm{Nm}^{2}$ \\
$E I_{y y}$ & Lateral bending stiffness & $6.32 \times 10^{9} \mathrm{Nm}^{2}$ \\
$G J$ & Longitudinal bending stiffness & $4.74 \times 10^{9} \mathrm{Nm}^{2}$ \\
\hline
\end{tabular}

The sample HSV is based on a 2D hypersonic cruise vehicle presented by Bolender and Doman ${ }^{1}$ and augmented with lifting surfaces introduced by Falkiewicz and Cesnik. ${ }^{18}$ The lifting surface dimensions are shown in Fig. 8. 

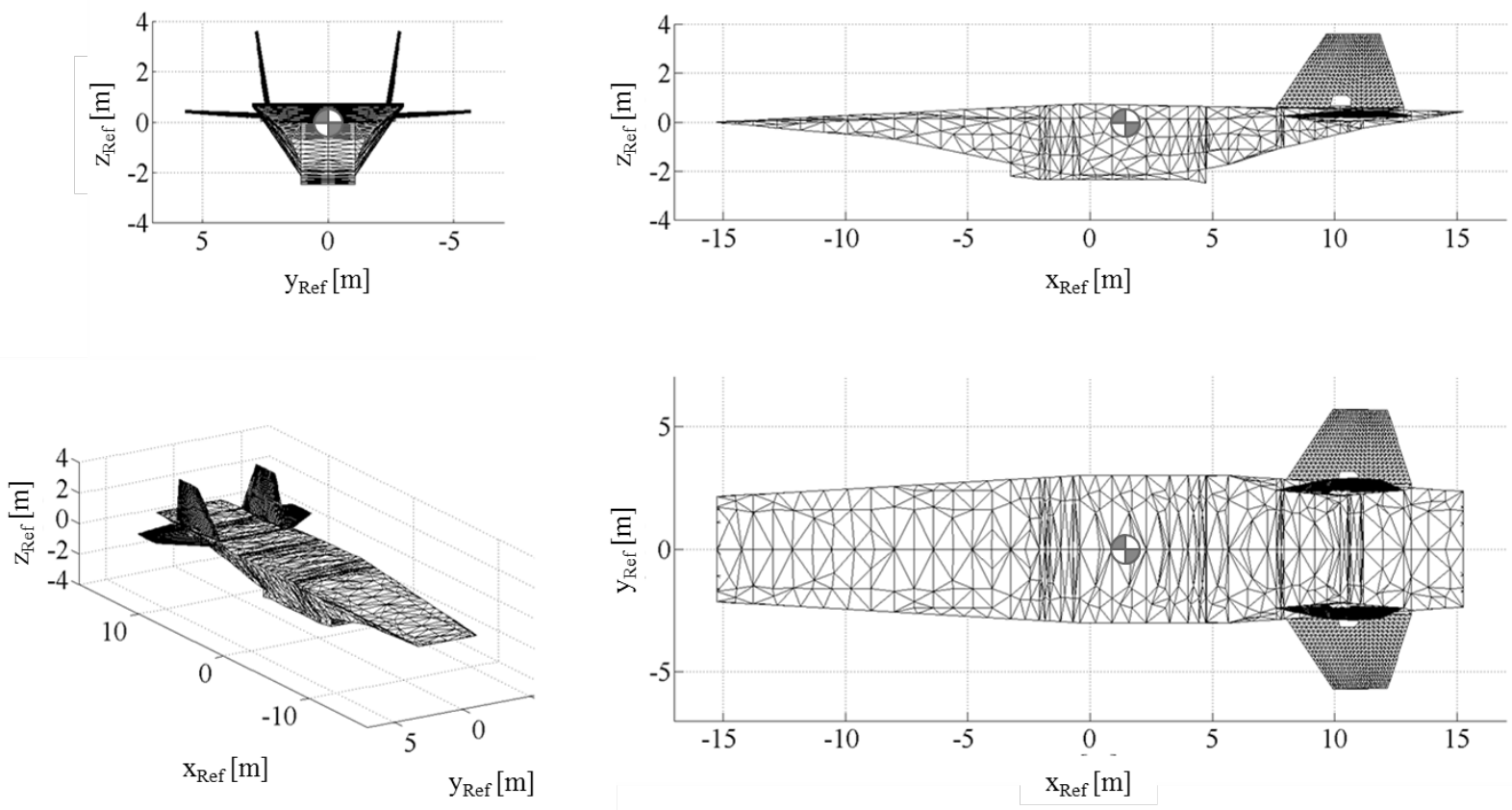

Figure 7: Sample HSV configuration

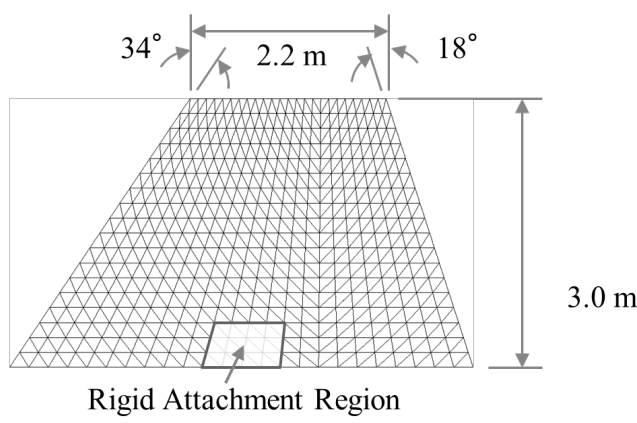

Figure 8: Basic lifting surface model (Ref. 18)

\section{B. Vehicle Trim}

Using the Matlab function fminsearch, the following cost function $J_{\text {trim }}$ can be minimized:

$$
J_{\text {trim }}=\left[\begin{array}{c}
\dot{\beta}_{x} \\
\dot{\beta}_{y} \\
\dot{\zeta}_{y} \\
\dot{\eta}_{y}
\end{array}\right]\left[\begin{array}{ll} 
\\
\end{array}\right]\left[\begin{array}{llll}
\dot{\beta}_{x} & \dot{\beta}_{y} & \dot{\zeta}_{y} & \dot{\eta}_{y}
\end{array}\right] \text {, }
$$

where the subscripts denote along which axes the generalized velocities $\dot{\beta}, \dot{\zeta}$, and $\dot{\eta}$ are considered and $I$ is the identity matrix. To avoid local minima, the solution of fminsearch is randomly perturbed by up to $10 \%$ for each parameter and reentered as the initial guess of another minimization. The two solutions are then compared for agreement. If the maximum residual is less than $1 \%$, the solution is considered to represent a global minimum. The 
trim conditions for both a rigid and flexible HSV considered in the sample simulation for steady level flight at Mach 6.0 and altitude of $26 \mathrm{~km}$ are given in Table 2.

Table 2: HSV steady level flight trimmed conditions for Mach 6, $26 \mathrm{~km}$ altitude

\begin{tabular}{|c|c|c|c|c|c|}
\hline \multirow[b]{2}{*}{ Symbol } & \multirow[b]{2}{*}{ Description } & \multicolumn{2}{|c|}{ Rigid Vehicle } & \multicolumn{2}{|c|}{ Flexible Vehicle } \\
\hline & & $\begin{array}{c}\text { Partitioned } \\
\text { Solution }\end{array}$ & $\begin{array}{l}\text { Monolithic } \\
\text { Solution }\end{array}$ & $\begin{array}{c}\text { Partitioned } \\
\text { Solution }\end{array}$ & $\begin{array}{l}\text { Monolithic } \\
\text { Solution }\end{array}$ \\
\hline$\alpha$ & Angle of attack (deg) & -0.64 & -0.61 & -0.66 & -0.61 \\
\hline$\eta_{1}$ & Lateral bending mode amplitude & - & - & 0 & 0 \\
\hline$\eta_{2}$ & $\begin{array}{l}\text { Longitudinal bending mode } \\
\text { amplitude }\end{array}$ & - & - & -0.003 & -0.004 \\
\hline$\eta_{3}$ & Torsion mode amplitude & - & - & 0 & 0 \\
\hline$\delta_{e}$ & Horizontal elevon deflection (deg) & 1.49 & 1.49 & 2.06 & 1.61 \\
\hline$\phi$ & Fuel equivalence ratio & 0.126 & 0.126 & 0.139 & 0.122 \\
\hline & Minimized Parameters & & & & \\
\hline$\dot{u}$ & Forward acceleration $\left(\mathrm{m} / \mathrm{s}^{2}\right)$ & $2.4 \times 10^{-1}$ & $1.6 \times 10^{-2}$ & $2.5 \times 10^{-1}$ & $3.1 \times 10^{-5}$ \\
\hline$\dot{w}$ & Vertical acceleration $\left(\mathrm{m} / \mathrm{s}^{2}\right)$ & $2.4 \times 10^{-2}$ & $1.5 \times 10^{-3}$ & $1.8 \times 10^{-2}$ & $-7.5 \times 10^{-5}$ \\
\hline$\dot{\omega}_{y}$ & Pitch angular acceleration $\left(\mathrm{deg} / \mathrm{s}^{2}\right)$ & $7.2 \times 10^{-3}$ & $4.9 \times 10^{-4}$ & $7.0 \times 10^{-3}$ & $9.2 \times 10^{-7}$ \\
\hline$\eta_{\text {lon }}$ & $\begin{array}{l}\text { Longitudinal bending mode } \\
\text { acceleration }\left(1 / \mathrm{s}^{2}\right)\end{array}$ & - & - & $9.2 \times 10^{-3}$ & $1.6 \times 10^{-5}$ \\
\hline
\end{tabular}

One can see from Table 2 that for the rigid vehicle cases, the partitioned and monolithic trim solutions match very well with only minor differences in the angle of attack and the minimization parameter values. This result supports that in steady cases the partitioned solution matches the monolithic solution. The flexible cases do not agree as well as the rigid ones, particularly for the elevon deflection angle. However, these disagreements are small, not exceeding 0.5 degrees difference between the elevon deflection angles and 0.02 between the fuel equivalence ratios. It is likely that these differences arose from the fact that the monolithic solution lifting surfaces are deformed slightly by the longitudinal bending mode of the fuselage. Since the entire vehicle has been considered as one object, this bending mode also applies to the lifting surface; whereas the partitioned solution lifting surfaces do not deform with the fuselage. A true monolithic model of this HSV representation would have to be created independently from the simulation architecture presented in this paper to eliminate these differences.

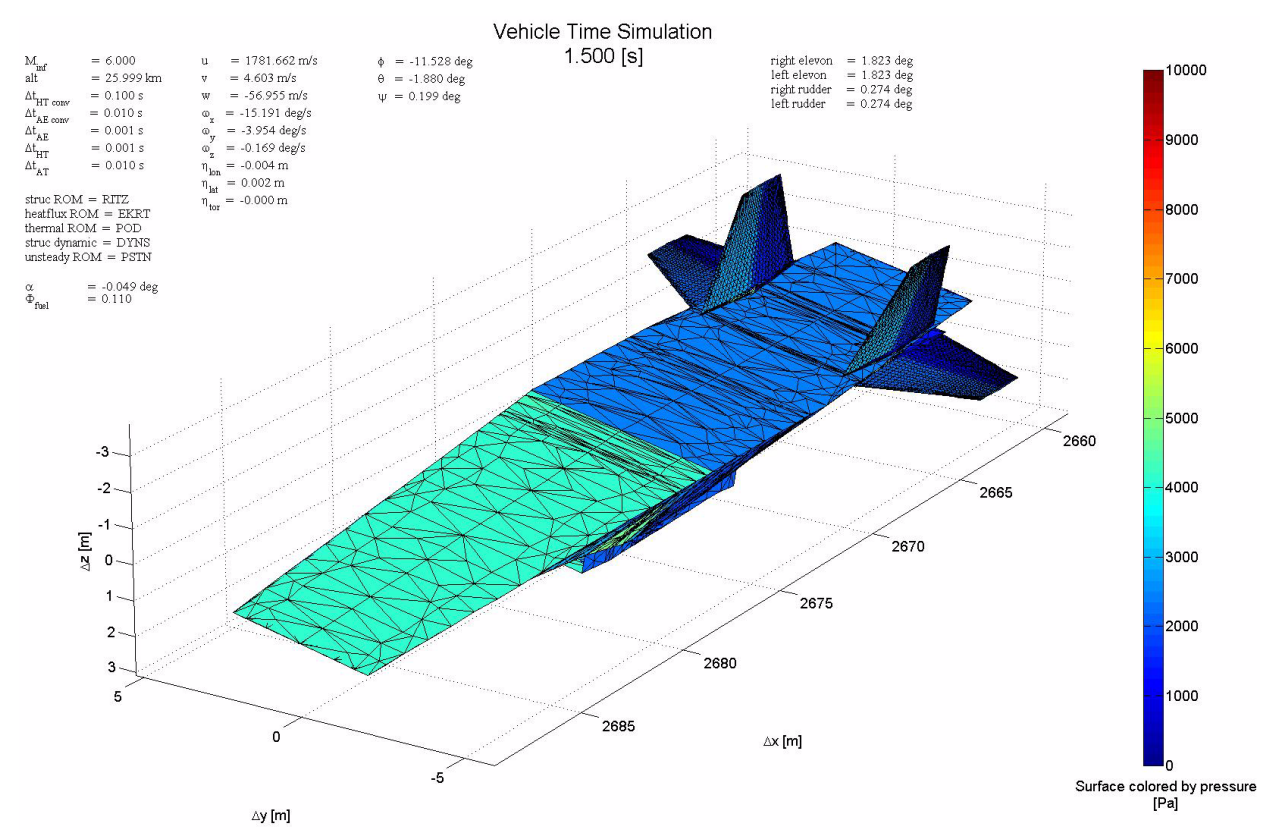

Figure 9: Sample HSV simulation video rendering during a roll maneuver

10 of 16

American Institute of Aeronautics and Astronautics 


\section{Time Simulation}

By iteratively computing the EOMs, component models, and generalized forces, the HSV simulation is marched forward in time. Time simulation results may be viewed as either video, as exemplified in Fig. 9, or time history plots as shown in Figs. 11 through 14. The partitioned and monolithic solutions are each simulated starting at their respective trim conditions outlined in Table 2 . The flight conditions and simulation parameters are listed in Table 3. Note that since the monolithic solution is treated as a main body object, the only time step of significance is the aeroelastic time step. The heat transfer time step, aerothermal time step, and residual displacement tolerance are only used for the partitioned solution.

After $0.5 \mathrm{~s}$ of flight at trim, each model is given a 1-degree elevon deflection command to induce a nose up pitch over the next $0.5 \mathrm{~s}$. The deflection is then held for $0.5 \mathrm{~s}$ after which the elevons return to their respective trim angles. The commanded elevon deflection for the rigid partitioned solution case over time is shown in Fig. 10.

Table 3: Sample HSV simulation parameters

\begin{tabular}{ccc}
\hline Parameter & Description & Value \\
\hline$M$ & Initial flight Mach number & 6.0 \\
$h$ & Initial flight altitude & $26.0 \mathrm{~km}$ \\
$\Delta t_{A E}$ & Aeroelastic time step & $0.001 \mathrm{~s}$ \\
$\Delta t_{H T}$ & Heat transfer time step & $0.001 \mathrm{~s}$ \\
$\Delta t_{A T}$ & Aerothermal time step & $0.010 \mathrm{~s}$ \\
$R_{t o l}$ & Fuselage/lifting surface residual displacement tolerance & $10^{-5} \mathrm{~m}$ \\
\hline
\end{tabular}

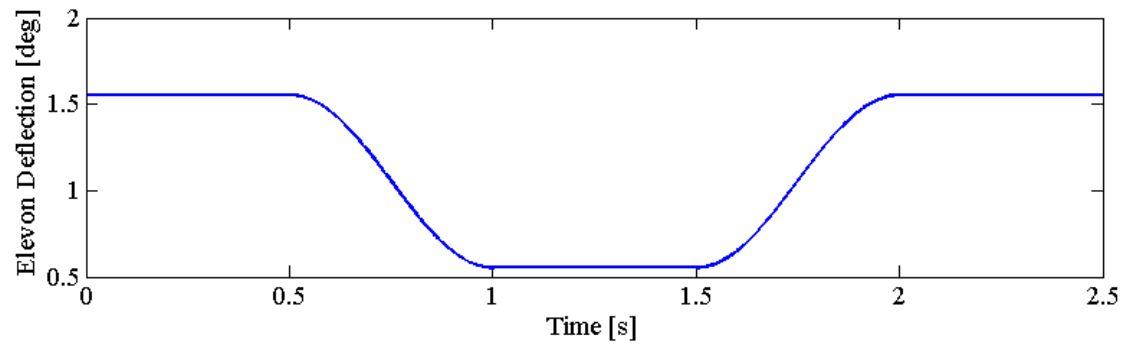

Figure 10: Commanded elevon deflection for the rigid partitioned solution case

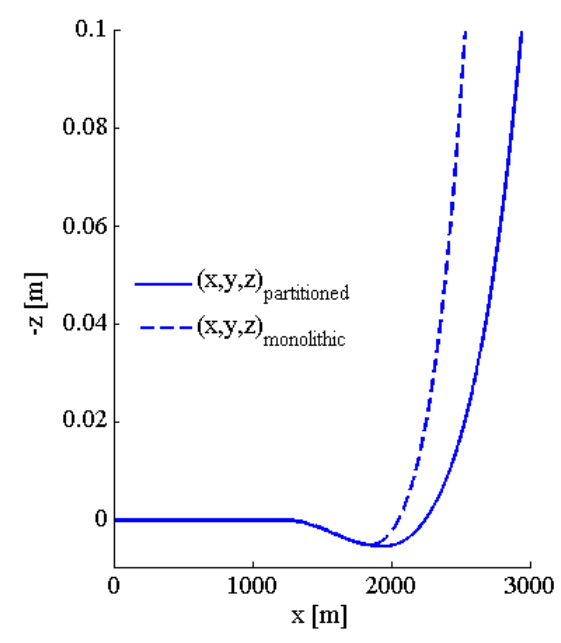

Figure 11: Rigid body position with respect to Earth frame due to elevon input of $+1^{\circ}$ (negative $z$ relates to increase in altitude)

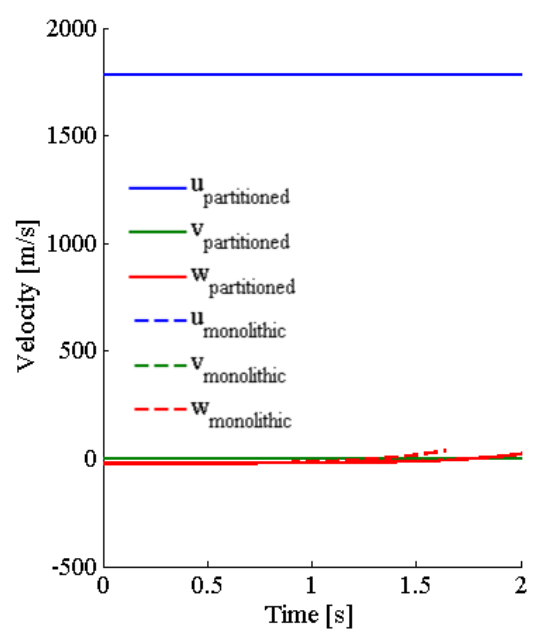

Figure 12: Rigid body velocity due to elevon input of $+1^{\circ}$

11 of 16 


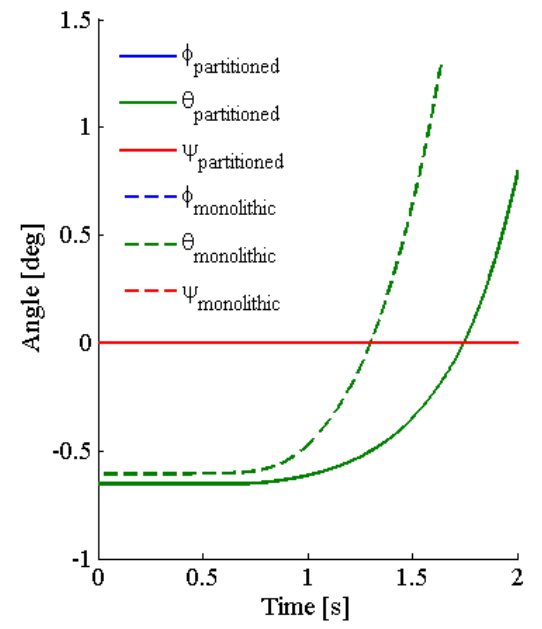

Figure 13: Rigid body Euler angles due to elevon input of $+1^{\circ}$

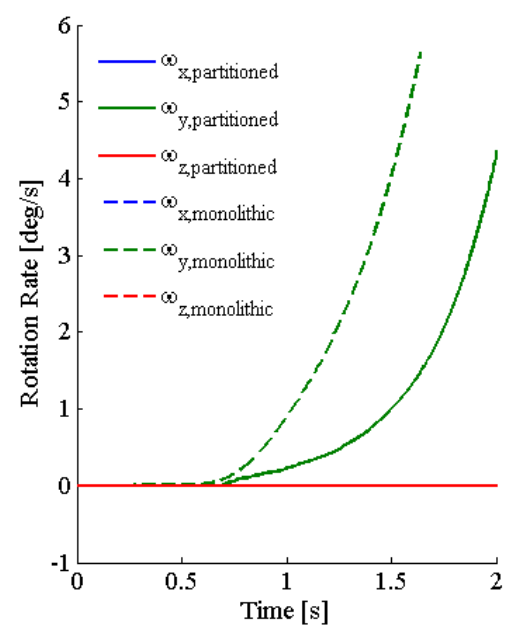

Figure 14: Rigid body angular rates due to elevon input of $+1^{\circ}$

Figures 11 through 14 show that the partitioned and monolithic solutions generally match in form and behavior. Figure 11 shows that each solution has captured the non-minimum phase behavior of the sample HSV as the elevons are first commanded to deflect at $0.5 \mathrm{~s}$. These figures also show that the monolithic vehicle solution was more responsive to the elevon deflection, pitching upward more quickly. This is due to a difference in mass distribution between the partitioned and monolithic models. The monolithic inertial model was treated as a distributed mass beam along the length of the model, neglecting the lumped mass of the lifting surfaces. The partitioned solution considered the masses of the lifting surfaces through the surfaces' EOMs. Thus the mass moment of inertia about the y-axis of the partitioned model was greater than the monolithic model. An adjustment to the monolithic model would have to be made to correct for the missing interface effects.

The HSV simulations were carried out on an Asus Workstation computer with an 8-core $2.00 \mathrm{GHz}$ Intel Xeon processor and 32 GB RAM. For 2 seconds of simulation, the partitioned solution was completed in $\sim 6$ hours and the monolithic solution in $\sim 10$ hours.

\section{Lifting Surface Stability Analysis}

Determining the aeroelastic stability of an HSV is critical in establishing the flight envelope of the vehicle. For the case of the basic vehicle model described in section IV.A., early time simulations at high Mach numbers (approximately Mach 8 or greater) and low altitude (approximately $10 \mathrm{~km}$ ) showed possible lifting surface flutter. To characterize the dynamic response of the lifting surfaces in flight a linear representation of the lifting surface motion can be established as

$$
\dot{x}(t)=A x(t),
$$

where $x(t)$ is the state vector, taken to be the amplitudes of the 15 structural Ritz modes described in section III.B. and their 15 respective time derivatives, and $A$ is the linear state matrix. The entries of $A$ are approximated from time simulations of the lifting surface as

$$
\begin{gathered}
\dot{x}=f(x), \\
A(:, k) \approx \frac{f\left(x_{e}+\Delta_{x k}\right)-f\left(x_{e}-\Delta_{x k}\right)}{2 \varepsilon_{x k}},
\end{gathered}
$$

where $A(:, k)$ denotes the $k^{\text {th }}$ column of $A, x_{e}$ is the trim state such that $f\left(x_{e}\right)=0$, and the perturbation $\Delta_{x k}$ is of the form 


$$
\Delta_{x k}=\left[\begin{array}{llllll}
0, & \cdots, & \varepsilon_{x k}, & 0, & \cdots, & 0
\end{array}\right]^{T},
$$

where $\varepsilon_{x k}$ is $1 \%$ of the $k^{\text {th }}$ trim state or a small value if the $k^{\text {th }}$ trim state is zero. The function $f\left(x_{e} \pm \Delta_{x k}\right)$ is approximated by a $6^{\text {th }}$-order forward difference of 7 time steps of the lifting surface simulation.

The structural properties of the lifting surface will vary over time due to the exposure to the hypersonic flow. Aerothermal heating of the lifting surface will degrade the material stiffness and uneven thermal expansion will create both geometric stiffening and thermal loads on the surface. To account for these effects, the solutions uses the Eckert reference temperature method and the POD thermal ROM to time march the thermal analysis of the lifting surface starting at the reference state of uniform 293-K structure. To decrease the flutter Mach number, the lifting surface is flown at $10 \mathrm{~km}$ altitude. Altitudes of 20 to $30 \mathrm{~km}$ are more typical for HSVs, however these would result in higher flutter Mach numbers and lower aeroheating, requiring longer simulation runtimes to heat the lifting surface sufficiently for thermal effects to make a similar impact.

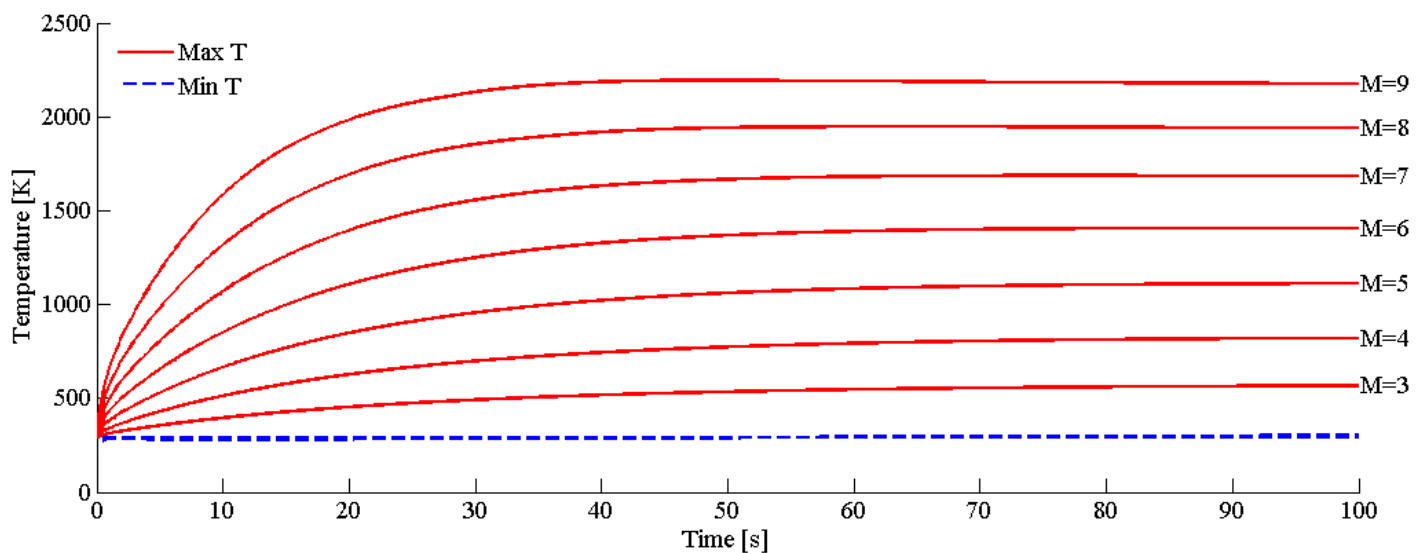

Figure 14: Temperature range of all points of the lifting surface structure for prolonged cruise at an altitude of $10 \mathrm{~km}$

The stability of the lifting surface at a given Mach number and time exposed to flow is determined by the relation of the eigenvalues $\lambda$ of $A$ of the form

$$
A v=\lambda v
$$

where $v$ are the associated eigenvectors. A mode of the lifting surface is deemed to be unstable when the corresponding root crosses to the positive real half plane. For the case of the lifting surface used as part of the sample HSV, started at a uniform $293 \mathrm{~K}$ and instantaneously exposed to the hypersonic flow, the behavior of the unstable (first vibration) mode is shown in Figs. 15 and 16. 


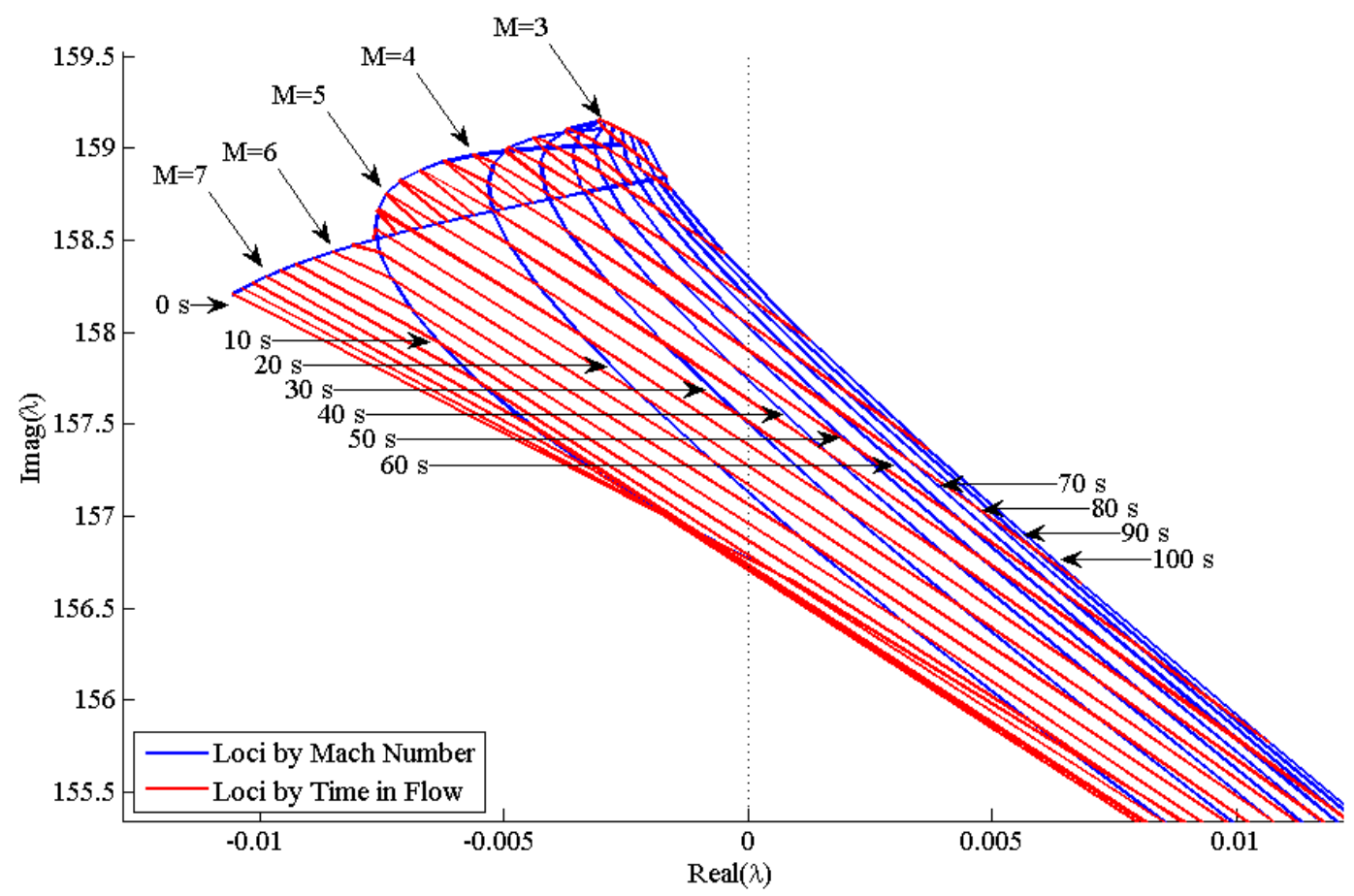

Figure 15: Root loci for various Mach numbers and flow exposure time for the first free vibration mode, $10 \mathrm{~km}$ altitude

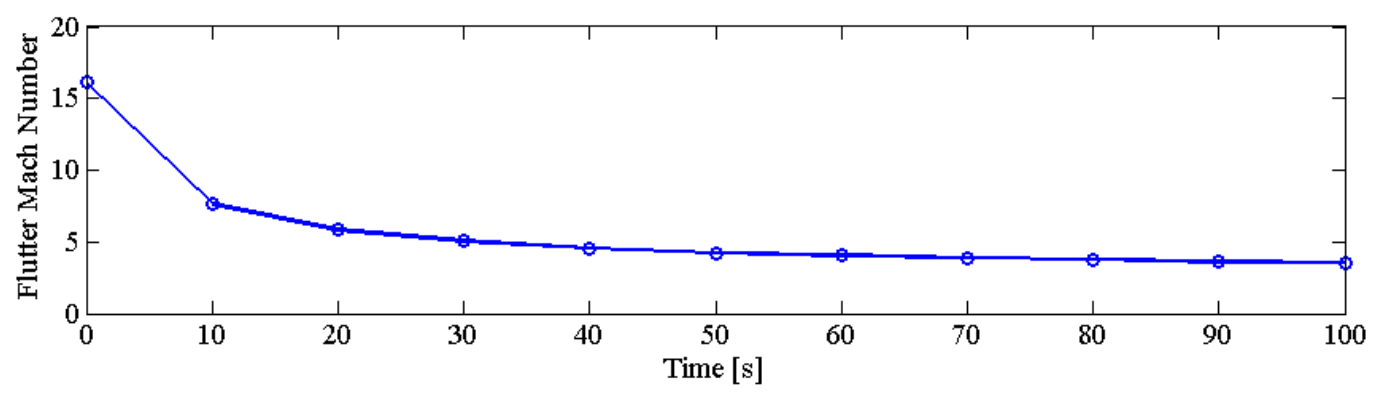

Figure 16: Time history of flutter Mach number, $10 \mathrm{~km}$ altitude

Figures 15 and 16 show that, as the lifting surface is first exposed to the flow, the lifting surface is relatively rigid and presents a flutter Mach number of 16.1. However as the lifting surface heats, there is a loss of stiffness, lowering the flutter Mach number. This loss of stiffness also lowers the frequency of the modes describing the elastic deformation of the lifting surface, corresponding to the negative slope of the loci by time in flow shown in Fig. 15. Also for lower Mach numbers, 3 though $\sim 5.5$, one can see a temporary increase in frequency likely due to geometric stiffening from the thermal gradients present of the lifting surface. As the lifting surface temperatures begin to stabilize after exposure to the flow, the flutter Mach number asymptotes to $\sim 3.5$, corresponding to a loss of over $78 \%$ of the initial flutter margin.

\section{Concluding Remarks}

This work presented the partitioned solution approach to simulation of an HSV. It considers the vehicle as partitioned into regions that independently model physical processes relevant to their performance and exchanges motion and force information across common interfaces at predetermined time intervals. This approach was implemented using a set of structural dynamics, unsteady aerodynamics, aeroheating, thermal, and propulsion ROMs previously developed for hypersonic analysis applications. The resulting simulation code architecture provides for solutions in HSV trim, time simulation, and aeroelastic stability analysis. The trim and time simulation analyses were compared to a monolithic approach by modeling a common sample HSV. Rigid vehicle trim results 
for Mach 6, 26-km altitude, steady level flight showed good agreement between the partitioned and monolithic solutions, with the greatest error between the angles of attack at less than $5 \%$. The flexible trim solutions did not agree as well, but errors remained small, not exceeding 0.5 -degrees difference between the elevon deflection angles and 0.02 between the fuel equivalence ratios. These were attributed to the difference in the baseline models used for each solution and not to the partitioned approach per se. For HSV trim, the partitioned solution agrees well with the monolithic solution.

Time simulation of the partitioned and monolithic solutions showed qualitative agreement, but differences arose due to a mismatch in the effective mass moment of inertia between the two models. This mismatch was due to the extra inertia derived from the separate lifting surface EOMs acting as lumped masses on the HSV main body.

Finally, a flutter margin analysis was conducted using the partitioned solution. Not unexpectedly, the flutter Mach number decreases significantly as aeroheating causes a reduction in the lifting surface stiffness. Flutter margin loss of over $78 \%$ is observed for $100-\mathrm{s}$ flight at $10-\mathrm{km}$ altitude, despite initial geometric stiffening of the lifting surface due to structural temperature gradients.

Overall, the partitioned approach presented an effective way to solve the multidisciplinary, multi-fidelity aerothermoelastic/propulsive hypersonic vehicle simulation problem.

\section{VI.Acknowledgements}

This work was supported by the Michigan-AFRL Collaborative Center in Control Science (MACCCS) under grant number FA 8650-07-2-3744 (Air Force Research Laboratory/Airspace Systems Directorate) and with Michael Bolender as technical monitor.

\section{References}

${ }^{1}$ Bolender, M.A. and Doman, D.B., "Nonlinear Longitudinal Dynamical Model of an Air-Breathing Hypersonic Vehicle," Journal of Spacecraft and Rockets, Vol. 44, No. 2, 2007, pp. 374-387.

${ }^{2}$ Chavez, F., Schmidt, D., "Analytical Aeropropulsive/Aeroelastic Hypersonic-Vehicle Model with Dynamics Analysis," Journal of Guidance, Control, and Dynamics, Vol. 17, No. 6, 1994, pp. 1308-1319.

${ }^{3}$ Frendreis, S.G.V., Skujins, T., and Cesnik, C.E.S., "Six-Degree-of-Freedom Simulation of Hypersonic Vehicles," Proceedings of the 2009 AIAA Atmospheric Flight Mechanics Conference, AIAA 2009-5601.

${ }^{4}$ Falkiewicz, N.J., Frendreis, S.G.V., and Cesnik, C.E.S., "Effect of Control Surface-Fuselage Inertial Coupling on Hypersonic Vehicle Flight Dynamics," Proceedings of 2011 AIAA Atmospheric Flight Mechanics Conference, AIAA 2011-6378.

${ }^{5}$ Falkiewicz, N.J., Cesnik, C.E.S., Crowell, A.R., and McNamara, J.J., "Reduced-Order Aerothermoelastic Framework for Hypersonic Vehicle Control Simulation," AIAA Journal, Vol. 49, No. 8, 2011, pp. 1625-1646.

${ }^{6}$ Falkiewicz, N.J. and Cesnik, C.E.S., "Proper Orthogonal Decomposition of Reduced-Order Thermal Solution in Hypersonic Aerothermoelastic Simulations," AIAA Journal, Vol. 49, No. 5, 2011, pp. 994-1009.

${ }^{7}$ Frendreis, S.G.V. and Cesnik, C.E.S., "3D Simulation of Flexible Hypersonic Vehicles," Proceedings of the 2010 AIAA Atmospheric Flight Mechanics Conference, AIAA 2010-8229.

${ }^{8}$ Dalle, D.J., Frendreis, S.G.V., Driscoll, J.F. and Cesnik, C.E.S., "Hypersonic Vehicle Flight Dynamics with Coupled Aerodynamics and Reduced-order Propulsive Models," Proceedings of the 2010 AIAA Atmospheric Flight Mechanics Conference, AIAA 2010-7930.

${ }^{9}$ Dalle, D.J. and Driscoll, J.F., "Continuous Differentiation of Complex Systems Applied to a Hypersonic Vehicle," Proceedings of the AIAA Atmospheric Flight Mechanics Conference, Minneapolis, Minnesota, AIAA 2012-4958.

${ }^{10}$ Anderson, J.D., Modern Compressible Flow with Historical Perspective, McGraw-Hill, 2002.

${ }^{11}$ Mahony, J.J., "A Critique of Shock-Expansion Theory," Journal of the Aeronautical Sciences, Vol. 22, No. 10, 1955, pp. 673-680.

${ }^{12}$ Jaslow, H., “Aerodynamic Relationships Inherent in Newtonian Impact Theory,” AIAA Journal, Vol. 6, No. 4, 1968, pp. 608-612.

${ }^{13}$ Ashely, H. and Zartarian, G., "Piston Theory - A New Aerodynamic Tool for the Aeroelastician," Journal of the Aeronautical Sciences, Vol. 23, No. 12, 1956, pp. 1109-1118.

${ }^{14}$ Skujins, T. and Cesnik, C.E.S., "Toward an Unsteady Aerodynamics ROM for Multiples Mach Regimes," Proceedings of the $53^{\text {rd }}$ AIAA/ASME/ASCE/AHS/ASC Structures, Structural Dynamics and Materials Conference, AIAA 2012-1708.

${ }^{15}$ Eckert, E.R.G., "Engineering Relations for Friction and Heat Transfer to Surfaces in High Velocity Flow," Journal of the Aeronautical Sciences, Vol. 22, No. 8, March 23, 1955, pp. 585-587.

15 of 16

American Institute of Aeronautics and Astronautics 
${ }^{16}$ Crowell, A.R. and McNamara, J.J., "Model Reduction of Computational Aerothermodynamics for Hypersonic Aerothermoelasticity," AIAA Journal, Vol. 50, No. 1, 2012, pp. 74-84.

${ }^{17}$ Torrez, S.M., Driscoll, J.F. and Dalle, D.J., "Scramjet Engine Model MASIV: Role of Mixing, Chemistry and Wave Interactions," Proceedings of the 45 $5^{\text {th }}$ AIAA/ASME/SAE/ASEE Joint Propulsion Conference \& Exhibit, Denver, Colorodo, AIAA 2009-4939.

${ }^{18}$ Falkiewicz, N.J. and Cesnik, C.E.S., "Enhanced Modal Solutions for Structural Dynamics in Aerothermoelastic Analysis," Proceedings of the 52 ${ }^{\text {nd }}$ AIAA/ASME/ASCE/AHS/ASC Structures, Structural Dynamics and Materials Conference, Denver, Colorado, AIAA 2011-1963. 